\title{
Nonlinear absorption in discrete systems
}

\author{
A. Spire and J. Leon \\ Physique Mathématique et Théorique, CNRS-UMR5825, \\ Université Montpellier 2, 34095 Montpellier (France)
}

\begin{abstract}
In the context of nonlinear scattering, a continuous wave incident onto a nonlinear discrete molecular chain of coupled oscillators can be partially absorbed as a result of a 3-wave resonant interaction that couples two $\mathrm{HF}$-waves of frequencies close to the edge of the Brillouin zone. Hence both nonlinearity and discreteness are necessary for generating this new absorption process which manisfests itself by soliton generation in the medium. As a paradigm of this nonlinear absorption we consider here the Davydov model that describes exciton-phonon coupling in hydrogen bonded molecular chains.
\end{abstract}

\section{Introduction}

The scattering of waves becomes extremely rich when nonlinearity comes into play, and one of its most fundamental effect is soliton generation which results in energy localization and propagation. For instance an incident radiation on a two-level medium at the resonant frequency can be totally transmitted, instead of being absorbed, a property described in [1] as self induced transparency. It results from the nonlinear coupling of radiation with medium population, a mechanism which generates the solitons, vectors of energy transmission.

Other interesting processes of nonlinear wave scattering are the two-photon propagation, second harmonic generation and stimulated Raman scattering. In that last case, the nonlinear interaction induces (laser) pump depletion and phase effects result in Raman spike generation (short duration pump repletion) 2]. Although the Raman spike generation is not a solitonic effect, here also the nonlinearity is the fundamental tool [3].

Recently discovered, the nonlinear supratransmission [4] 5] is a nonlinear scattering process where a totally reflective medium switches to high transmissivity above some threshold because of nonlinearity. The bifurcation takes its origin in a nonlinear instability [6] which has a wide field of application in generic nonlinear evolutions [7].

Particularly interesting is the application of this concept to the scattering of a continuous-wave laser beam incident onto a Bragg mirror which switches from total reflection to transmission by means of gap soliton generation [8, 9]. 
It has been demonstrated that the switch is a manifestation of nonlinear supratransmission which has in particular allowed to compute an analytic explicit expression of the intensity flux threshold [10].

We are interested here in the concept of nonlinear absorption where a medium, transparent to incident radiation in the linear regime, can become absorbant under contribution of nonlinearity which is due here to coupling of waves of different nature. Such is the case with the Davydov model [1] describing the coupling of high frequency optical phonon waves to low frequency acoustic waves. A generic field of application of the Davydov model is the cristallin acetanilide where the acoustic wave represents the hydrogen bond between adjacent molecules, while the optical phonon wave represents the $\mathrm{C}=\mathrm{O}$ stretching (amide-I mode). This model has been widely studied in its stationnary limit where the hydrogen bond is supposed to have a time response much longer than the high frequency $\mathrm{C}=\mathrm{O}$ stretching. In that situation the resulting simplified model is the nonlinear Schrödinger equation that has been used to interprete the anomalous IR-absorption band by soliton generation [12].

The Davydov model has been recently reexamined in the context of resonant two-wave interaction where a rigorous multiscale analysis transforms the discrete model to a continuous integrable partial differential equation which keeps the fundamental wave coupling effect and possess multi-soliton solutions 13. This two-wave resonant interaction process takes into account an incident high-frequency optical phonon wave that resonates with the low-frequency acoustic wave as soon as the HF-wave group velocity equals the LF-wave phase velocity. This is the Benney criterion [14] that has revealed its efficiency through numerical simulations of the Davydov model [15.

The two-wave resonant process assumes no backward propagation and thus neglects the multiple reflections due to the periodicity of the cristal. We consider here a three-wave interaction process that couples the incident optical phonon wave both to the acoustic excitation and the reflected optical phonon wave. We shall discover that an incident and a reflected high-frequency waves can cooperate resonantly with a low-frequency acoustic wave thanks to the discrete nature of the basic model.

We will demonstrate that this scattering process allows for absorption of incident radiation by a purely nonlinear effect that generates a three-wave soliton in the medium. The nonlinear effect is effectively an absorption as a part of the incident energy flux is transfered to the medium.

\section{Three-wave interaction in the Davydov model}

\section{$2.1 \quad$ Basic model}

Our starting point is the Davydov model 11 for the eigenstate $a_{n}(t)$ of the amide-I excitation (the corresponding dynamical variable represents the $\mathrm{C}=\mathrm{O}$ stretching) coupled to the dynamical variable $\beta_{n}$ that represents the longitudinal 
displacement along the hydrogen-bond chain. It reads

$$
\begin{aligned}
& i \hbar \dot{a}_{n}=\left[E_{0}+W+\chi\left(\beta_{n+1}-\beta_{n}\right)\right] a_{n}-J\left(a_{n+1}+a_{n-1}\right), \\
& M \ddot{\beta}_{n}=K\left(\beta_{n+1}-2 \beta_{n}+\beta_{n-1}\right)+\chi\left(\left|a_{n}\right|^{2}-\left|a_{n-1}\right|^{2}\right),
\end{aligned}
$$

where $M$ is the mass of the peptide group, $K$ the spring constant of the hydrogen bond, $E_{0}$ the energy of the $\mathrm{C}=\mathrm{O}$ stretching and $W$ the the total energy of the peptide group displacements. The constant $\chi$ is the coupling parameter and $J$ measures the energy of the dipole-dipole interaction of $\mathrm{C}=\mathrm{O}$ stretching oscillations. Overdot stands for derivation with respect to the physical time $T$.

Upon defining the dimensionless time $t=J T / \hbar$, and the adimensional quantities

$$
\Psi_{n}=a_{n} \frac{\hbar \chi}{J \sqrt{J M}} \exp \left[\frac{i}{J}\left(E_{0}+W-2 J\right) t\right], \quad Q_{n}=\frac{\chi}{J}\left(\beta_{n+1}-\beta_{n}\right),
$$

the system becomes

$$
\begin{aligned}
& i \partial_{t} \Psi_{n}+\left(\Psi_{n+1}-2 \Psi_{n}+\Psi_{n-1}\right)=Q_{n} \Psi_{n}, \\
& \partial_{t}^{2} Q_{n}-V^{2}\left(Q_{n+1}-2 Q_{n}+Q_{n-1}\right)=\left|\Psi_{n+1}\right|^{2}-2\left|\Psi_{n}\right|^{2}+\left|\Psi_{n-1}\right|^{2},
\end{aligned}
$$

which constitutes our basic example of a discrete nonlinear coupled wave system of equations. Note that we are left with a single constant, the adimensional sound velocity

$$
V=\frac{\hbar}{J} v_{p}, \quad v_{p}=\sqrt{\frac{K}{M}},
$$

where $v_{p}$ the phonon velocity (cells per second), and that the coupling parameter $\chi$ has been absorbed in the amplitude of $\psi_{n}(t)$.

\subsection{Multi-scale expansion}

Following the multiscale expansion method [16] we assume a representation of the solution $\left\{\Psi_{n}(t), Q_{n}(t)\right\}$ as formal series in terms of a small parameter $\epsilon$ where space-time dependences occur at a sequence of slow scales. Since it is not necessary to push the series at arbitrary order, we write explicitely the first relevant terms only as

$$
\begin{aligned}
& \Psi_{n}(t)=\epsilon \varphi^{(0)}\left(n_{0}, x_{1}, \cdots ; t_{0}, t_{1}, \cdots\right)+\epsilon^{2} \varphi^{(1)}\left(n_{0}, x_{1}, \cdots ; t_{0}, t_{1}, \cdots\right)+\cdots \\
& Q_{n}(t)=\epsilon q^{(0)}\left(n_{0}, x_{1}, \cdots ; t_{0}, t_{1}, \cdots\right)+\epsilon^{2} q^{(1)}\left(n_{0}, x_{1}, \cdots ; t_{0}, t_{1}, \cdots\right)+\cdots
\end{aligned}
$$

and the difference-differential operators must be understood as

$$
\nabla_{n}^{ \pm} \rightarrow \nabla_{n_{0}}^{ \pm}+\epsilon \partial_{x_{1}}+\cdots, \quad \partial_{t} \rightarrow \partial_{t_{0}}+\epsilon \partial_{t_{1}}+\cdots
$$

Hereabove the difference operators are defined as

$$
\nabla_{n}^{+} \Psi_{n}=\Psi_{n+1}-\Psi_{n}, \quad \nabla_{n}^{-} \Psi_{n}=\Psi_{n}-\Psi_{n-1},
$$


such that the second order difference appearing in (4) is

$$
\nabla_{n}^{2} \Psi_{n}=\nabla_{n}^{+} \nabla_{n}^{-} \Psi_{n}=\Psi_{n+1}+\Psi_{n-1}-2 \Psi_{n} .
$$

The system (45) at first order gives the linear equations

$$
\begin{array}{ll}
\mathcal{L}_{0} \varphi^{(0)}=0, & \mathcal{L}_{0}=i \partial_{t_{0}}+\nabla_{n_{0}}^{2} \\
L_{0} q^{(0)}=0, & L_{0}=\partial_{t_{0}}^{2}-V^{2} \nabla_{n_{0}}^{2}
\end{array}
$$

The next order $\epsilon^{2}$ eventually reads

$$
\begin{aligned}
& \mathcal{L}_{0} \varphi^{(1)}=-i \partial_{t_{1}} \varphi^{(0)}-\left(\nabla_{n_{0}}^{+}+\nabla_{n_{0}}^{-}\right) \partial_{x_{1}} \varphi^{(0)}+q^{(0)} \varphi^{(0)} \\
& L_{0} q^{(1)}=2 \partial_{t_{0}} \partial_{t_{1}} q^{(0)}+V^{2}\left(\nabla_{n_{0}}^{+}+\nabla_{n_{0}}^{-}\right) \partial_{x_{1}} q^{(0)}+\nabla_{n_{0}}^{2}\left|\varphi^{(0)}\right|^{2} .
\end{aligned}
$$

The method then works as follows. Once selected an explicit solution of the linear system (12), we express that the evolution (13) of the first order correction $\left\{\varphi_{n}^{(1)}, q_{n}^{(1)}\right\}$ must not produce secular growth. This explicitely furnishes the evolution of the fundamental $\left\{\varphi_{n}^{(0)}, q_{n}^{(0)}\right\}$ in the slow variables $x_{1}$ and $t_{1}$. The choice of the linear solution in the variables $n_{0}, t_{0}$ determines the physical problem under study.

\subsection{Selection rules}

To describe a 3-wave interaction process involving incident and backscattered optical phonon waves, we are led to select in the linear system (12) the solution

$$
\begin{aligned}
& \varphi^{(0)}=A\left(x_{1}, t_{1}\right) e^{i\left(k_{1} n_{0}-\omega_{1} t_{0}\right)}+B\left(x_{1}, t_{1}\right) e^{i\left(-k_{2} n_{0}-\omega_{2} t_{0}\right)}, \\
& q^{(0)}=g\left(x_{1}, t_{1}\right) e^{i\left(K n_{0}-\Omega t_{0}\right)}+\bar{g}\left(x_{1}, t_{1}\right) e^{-i\left(K n_{0}-\Omega t_{0}\right)},
\end{aligned}
$$

with wave numbers $k_{1}>0$ and $k_{2}>0$ such as to ensure an optical phonon wave as a superposition of an incomming wave of amplitude $A$ and a reflected wave of amplitude $B$. The acoustic wave can propagate in both directions, thus $K$ can be of either signs. We have explicitely written the variables $\left(x_{1}, t_{1}\right)$ in the slowly varying amplitudes $A, B$, and $g$, but of course they depend on all higher order variables (but not on the first order ones $n_{0}$ and $t_{0}$ ). The above expression is a solution of (12) for the following dispersion relations

$$
\omega_{1}=2\left(1-\cos k_{1}\right), \quad \omega_{2}=2\left(1-\cos k_{2}\right), \quad \Omega=2 V\left|\sin \frac{K}{2}\right| .
$$

Note that using the relation (3) and the multiscale derivative laws, we demonstrate that the first order enveloppe $\beta_{0}$ of the longitudinal displacement along the hydrogen-bond chain $\beta_{n}$ is related to the enveloppe $g\left(x_{1}, t_{1}\right)$ of $Q_{n}$ by

$$
\beta_{0}\left(x_{1}, t_{1}\right)=-i \frac{J}{2 \chi \sin (K)} g\left(x_{1}, t_{1}\right)
$$


then the behaviour of the enveloppe $g\left(x_{1}, t_{1}\right)$ will automatically gives the behaviour of $\beta_{0}\left(x_{1}, t_{1}\right)$.

The resonant wave interaction results from a selection rule for the wave parameters, obtained by examination of the nonlinear terms that occur in (13), namely

$$
\begin{aligned}
q^{(0)} \varphi^{(0)}= & A g e^{i\left(k_{1}+K\right) n_{0}} e^{-i\left(\omega_{1}+\Omega\right) t_{0}}+A \bar{g} e^{i\left(k_{1}-K\right) n_{0}} e^{-i\left(\omega_{1}-\Omega\right) t_{0}} \\
& +B g e^{i\left(-k_{2}+K\right) n_{0}} e^{-i\left(\omega_{2}+\Omega\right) t_{0}}+B \bar{g} e^{i\left(-k_{2}-K\right) n_{0}} e^{-i\left(\omega_{2}-\Omega\right) t_{0}} .
\end{aligned}
$$

Such terms will combine to either components of $\varphi^{(0)}$ and resonate with corresponding factors in the left-hand-side of (13). The evolution of the envelopes will be then obtained by setting to zero the coefficients of resonating terms.

Since the physical context is the resonant interaction of two HF-waves (optical phonon) with a LF acoustic wave, it implies a small value of $K$ (near the center of the Brillouin zone) and large values of $k_{1}$ and $k_{2}$, that is to say close to, but less than, the value $\pi$ in order to achieve incident and reflected HF-waves. We are then left with the following selection rules. First when the nonlinear terms $A g$ and $B \bar{g}$ combine respectively with $B$ and $A$ we get

$$
k 1+k 2=2 \pi-K, \quad K>0, \quad \omega_{1}-\omega_{2}=-\Omega,
$$

Another possibility is to combine instead $A \bar{g}$ and $B g$ with $B$ and $A$ to obtain

$$
k 1+k 2=2 \pi+K, \quad K<0, \quad \omega_{1}-\omega_{2}=\Omega .
$$

We shall study these two cases and demonstrate that only the first one gives an instability that generates solitons, it is displayed on fig. 1

It is worth remarking that such a scattering process (involving $2 \pi$ ) is allowed for by the presence of an exponential in the discrete variable $\left(n_{0}\right)$, in other words by the discrete nature of the medium.
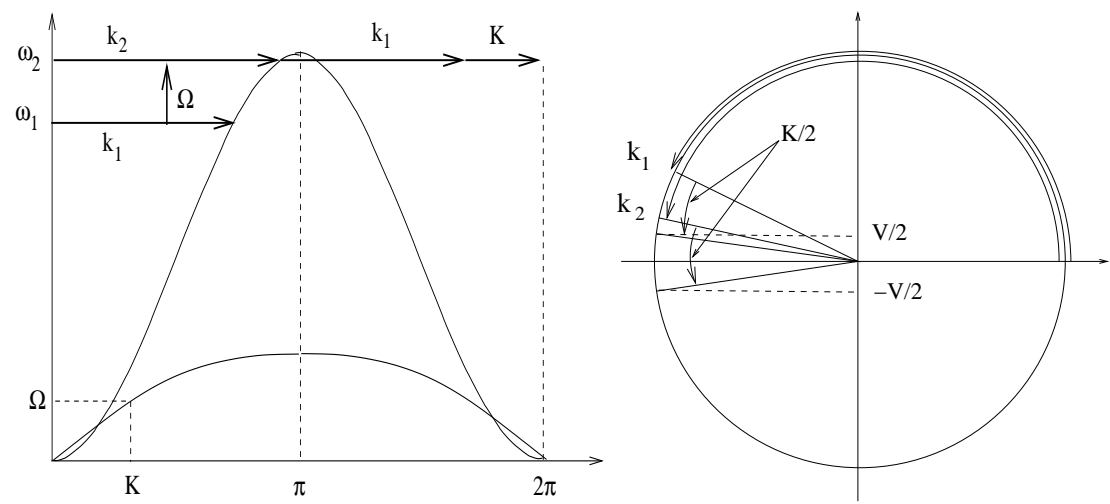

Figure 1: Representation of the selection rules (18) $(V=0.5)$ and graph of the solution (20). 
Inserting the dispersion relations (16) in the equation (18), we obtain after some algebra the following solution

$$
2 \sin \left(k_{1}+K / 2\right)=V, \quad 2 \sin \left(k_{2}+K / 2\right)=-V .
$$

These equations determine completely the wave nubers $k_{2}$ and $K$ from the data of $V$ (physics) and $k_{1}$ (incident wave), as soon as one assumes that $k_{1}$ an $k_{2}$ are close to, and less than $\pi$.

Remark : a particular case is obtained in the limit $K \rightarrow 0$ for which $k_{2} \rightarrow$ $-k_{1}$ and $2 \sin \left(k_{1}\right)=V$. This is the two-wave resonant interaction studied in [13] for which a different multi-scale analysis has to be employed (resonant process occurs at larger scales).

\subsection{Three-wave resonant interaction}

Corresponding to the first selection rule (18), the evolution equations for the envelopes that ensure vanishing of the resonant terms in the evolution (13) read

$$
\begin{aligned}
& {\left[\partial_{t}+2 \sin k_{1} \partial_{x}\right] A=-i B \bar{g},} \\
& {\left[\partial_{t}-2 \sin k_{2} \partial_{x}\right] B=-i A g,} \\
& {\left[\partial_{t}-V^{2} \frac{\sin K}{\Omega} \partial_{x}\right] g=i \frac{\Omega}{2 V^{2}} B \bar{A} .}
\end{aligned}
$$

From now on we rename $x_{1}$ by $x$ and $t_{1}$ by $t$. The same procedure applied to the second selection rule (19) eventually furnishes

$$
\begin{aligned}
& {\left[\partial_{t}+2 \sin k_{1} \partial_{x}\right] A=-i B g,} \\
& {\left[\partial_{t}-2 \sin k_{2} \partial_{x}\right] B=-i A \bar{g}} \\
& {\left[\partial_{t}-V^{2} \frac{\sin K}{\Omega} \partial_{x}\right] g=i \frac{\Omega}{2 V^{2}} A \bar{B} .}
\end{aligned}
$$

These are two standard 3-wave interaction nonlinear evolutions which are integrable systems on the infinite line $x \in \mathbb{R}$ when Cauchy initial data are prescribed in a space of functions vanishing (together with all their derivatives) at $x \rightarrow \pm \infty$ 17.

Such an initial-boundary value problem is not the one we are interested in as indeed we study the scattering of an incident $\mathrm{HF}$ wave of envelope $A(x, t)$ having a prescribed value for all time $t$ at the origin of the medium, i.e. at $x=0$. Moreover, as $B(x, t)$ stands for the envelope of the reflected wave, its value will be prescribed to vanish at the output $x=L$ for all time. Thus we cannot make use of the inverse scattering transform, unless first reformulated for a boundary-value problem on the finite interval, which is still an open problem.

Note that the system (22), by renaming $g$ as $\bar{g}$, maps to the first one except for the sign of the inhomogeneous term of the last equation. This change of sign is fundamental as it switches from instability to stability, as described below. 


\subsection{Stability properties}

The problem we consider is thus the scattering of an incident HF wave of envelope $A(x, t)$, belonging to a carrier wave of frequency $\omega_{1}$, that generates backscattered wave of envelope $B(x, t)$ and LF acoustic wave of envelope $g(x, t)$ out of initial vacuum. Thus we perform a linear stability analysis of both systems about the solution

$$
A(x, t)=A_{c}, \quad B=0, \quad g=0 .
$$

with constant $A_{c}$ corresponding to a continuous wave (CW) irradiation. Mathematically speaking this is an exact solution of both systems (21) and (22) and thus only an instability could produce an effective scattering. Let us seek now a solution as the perturbation

$$
A=A_{c}+\epsilon a e^{-i \nu t}, \quad B=\epsilon b e^{-i \nu t}, \quad g=\epsilon q e^{-i \nu t} .
$$

The system (21) at order $\epsilon$ then gives the linear system

$$
\left(\begin{array}{ccc}
\nu & 0 & 0 \\
0 & \nu & A_{c} \\
0 & -\bar{A}_{c} \frac{\Omega}{2 V^{2}} & \nu
\end{array}\right)\left(\begin{array}{l}
a \\
b \\
q
\end{array}\right)=0
$$

possessing the 3 eigenvalues $\nu=0$ and $\nu= \pm i\left(\left|A_{c}\right| / V\right) \sqrt{\Omega / 2}$. The system is thus unstable $(\Omega>0)$, while the same analysis with system (22) and $g=\epsilon q e^{i \nu t}$, furnishes the real eigenvalues 0 and $\pm\left(\left|A_{c}\right| / V\right) \sqrt{\Omega / 2}$, and thus stability.

Consequently the linear stability analysis predicts that the selection rule (18) will produce an effective scattering for an incident CW wave. Our purpose now is to demonstrate by numerical simulations that this instability is a soliton generator which induces an effective energy absorption of the incident radiation.

\section{$3 \quad$ Numerical simulations}

In order to understand the effect of the instability of the solution (23) in system (21), and compare it to system (22), we perform here numerical simulations of those sytems under the following initial-boundary value data on the interval $x \in[0, L]$,

$$
A(0, t)=A_{c}, \quad B(L, t)=0, \quad g(x, 0)=0 .
$$

As an illustartion the figure 2 shows the energy density profile $|A(x, t)|^{2}$ of the incident wave as a function of $x$ at time $t=20$ for $A_{c}=0.7$. The dashed line represents the acoustic phonon $|g(x, t)|^{2}$ that has gained the energy lost by the incident optical phonon.

This process corresponds to a brutal transfer of energy from the incident wave (which has already settled in the medium) to the medium, as shown on fig. 3 The normalized energy carried by the waves of envelopes $A$ and $g$ are 


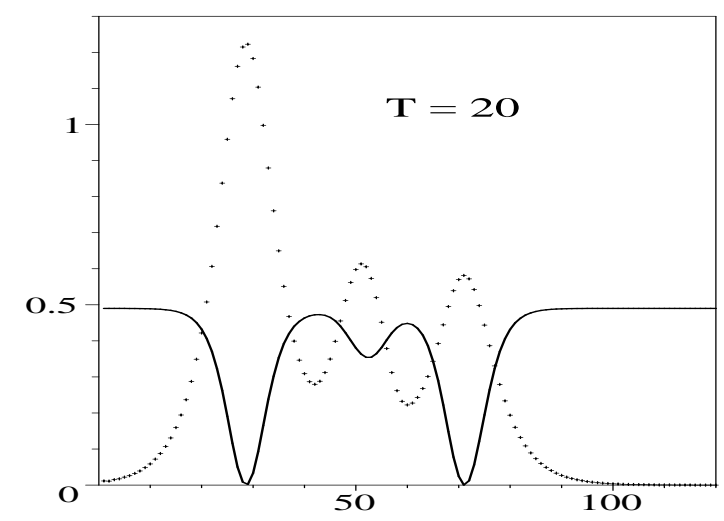

Figure 2: Energy density at time $t=20$ for optical phonon wave $|A(x, t)|^{2}$ (full line) and $|g(x, t)|^{2}$ (cross).

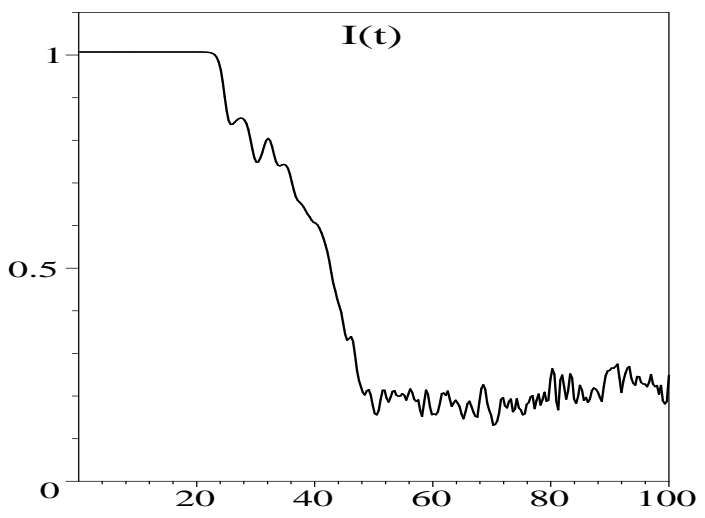

Figure 3: Energy of the optical phonon wave $I(t)$ for $A_{c}=0.7$ and the values (29)

defined by

$$
\begin{aligned}
& I(t)=\frac{1}{L\left|A_{c}^{2}\right|^{2}} \int_{0}^{L} d x|A(x, t)|^{2}, \\
& P(t)=\frac{1}{L\left|A_{c}^{2}\right|^{2}} \int_{0}^{L} d x|g(x, t)|^{2} .
\end{aligned}
$$

These are the quantities evaluated on a numerical simulation in fig. 3 for the following parameters

$$
\begin{aligned}
& \omega_{1}=3.94, \quad k_{1}=2.89 \\
& \omega_{2}=3.98, \quad k_{2}=2.99 \\
& V=0.1, \quad \Omega=0.04, \quad K=0.4
\end{aligned}
$$


Performing the same numerical simulations in the case of the system (22) have never produced any nonlinear energy transfer, confirming the predictions of the linear stability analysis.

\section{Conclusion}

We have shown that a molecular chain allowing for wave coupling process, like the Davydov model, can present nonlinear energy absorption by resonant interaction, coupling HF to LF waves, with selection rules (18) that are allowed only in discrete systems.

The resulting governing equation, though being integrable (for a Cauchy initial value problem on the infinite line) leads to an instability when driven by boundary data on the finite interval. This instability is then the source of soliton formation in the medium, and energy transfers from the incident radiation to the medium excitation.

This process is clearly illustrated by numerical simulations and opens the way to further studies in different contexts where the discreteness is known $a$ priori to play a fundamental role.

\section{References}

\section{References}

[1] S.L. Mc Call, E.L. Hahnn, Phys Rev 183 (1969) 457

[2] K. Drühl, R.G. Wenzel, J.L. Carlsten, Phys Rev Lett 51 (1983) 1171

[3] C. Claude, J. Leon, Phys Rev Lett 74 (1995) 3479. J. Leon, A.V. Mikhailov, Phys Lett A 253 (1999) 33

[4] J-G. Caputo, J. Leon, A. Spire, Phys Lett A 283 (2001) 129

[5] F. Geniet, J. Leon, Phys Rev Lett 89 (2002) 134102

[6] J. Leon, Phys Lett A 319 (2003) 130

[7] F. Geniet and J. Leon, J Phys: Condens Matter 15 (2003) 2933

[8] A. Yariv, Optical Electronics, Saunders Collge Pub. (4th edition), Orlando, FL-USA (1991)

[9] N.D. Sankey, D.F. Prelewitz, T.G. Brown, Appl Phys Lett 60 (1992) 1427

[10] J. Leon, A. Spire, to appear in Phys. Lett. A http://arxiv.org/abs/nlin/0310006

[11] A.S. Davydov, Solitons in molecular systems, D. Reidel Publishing Compagny, (Dordrecht 1985) 
[12] A.C. Scott, Phys Reports 217 (1992) 1

[13] M. Boiti, J. Leon , F. Pempinelli, A. Spire, J Phys A : Math Gener 37 (2004) 4243

[14] D.J. Benney, Stud appl math 56 (1977) 81

[15] G. Kopidakis, C.M. Soukoulis, E.N. Economou, Phys Rev B 51 (1995) 15038

[16] G.B Whitham, Linear and nonlinear waves, Wiley-Interscience, John Wiley \& Sons (New York, 1974)

[17] D.J. Kaup, Stud Appl Math 55 (1976) 9 